\title{
Pushing the complexity barrier: diminishing returns in the sciences
}

\author{
Claudius Gros \\ Institute for Theoretical Physics, \\ J.W. Goethe University Frankfurt, Germany
}

September 17, 2012

Are the sciences not advancing at an ever increasing speed? We contrast this popular perspective with the view that scientific research is actually closing in to complexity barriers and that, as a consequence, science funding actually sees diminishing returns, at least in established fields. In order to stimulate a larger discussion, we investigate two exemplary cases, the linear increase in human life expectancy over the last 170 years and the advances in the reliability of numerical short and medium term weather predictions during the last 50 years. We argue that the outcome of science and technology funding in terms of measurable results is a highly sub-linear function of the amount of resources committed. Supporting a range of small to medium size research projects, instead of a few large ones, will be, as a corollary, a more efficient use of resources for science funding agencies.

\section{Measuring scientific progress}

There is a curious dichotomy in our current science and technology (S\&T) landscape. On one side we see advances on scales as never before in human history. There is, on the other side, a growing sentiment among researchers that progress in science is becoming increasingly harder to achieve. This sentiment is based in part on anecdotal evidences and is continuously reinforced by new insights. On the anecdotal side there is the phenomenon that the requirements for a typical $\mathrm{PhD}$ thesis in the natural sciences have increased dramatically over the last 50 years. It is well known that it takes nowadays much longer for a young scientist to reach the forefront of research.

The notion that scientific research needs to deal with raising levels of complexity is especially evident when studying the realm of life. A paradigmal example of new insights bolstering this notion are the results of the ENCODE project [1, showing that our genome does not only contain 21 thousand protein encoding genes, but also up to 4 million regulatory switches where transcription factors could bind, besides a myriad of other regulatory sequences. Is it possible to quantify this notion of a raising complexity level? This is the central topic of our investigation and it involves the quest to actually measure the pace of scientific progress.

Scientific progress is notoriously hard to measure. It doesn't really make sense to quantify advances in fundamental research; a single publication leading to a paradigm shift may be invaluable. The vast majority of scientific research efforts are however directed towards achieving incremental 


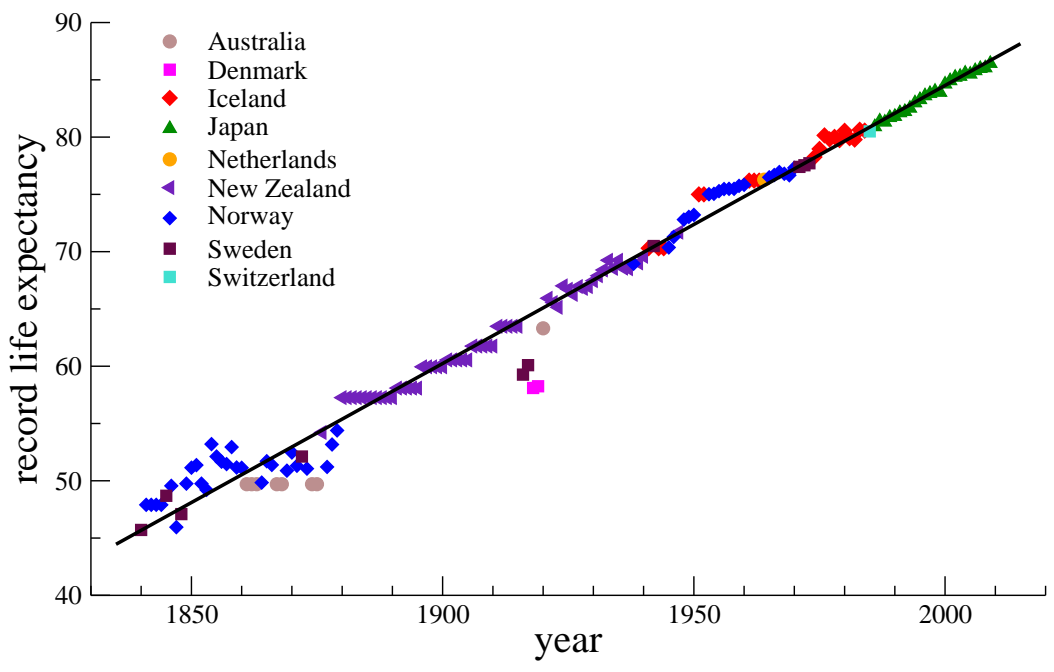

Figure 1: Record female life expectancy [4, 5. Shown is the life expectancy of the country with the highest average female life expectancy in a given year, for all calendar years. The line is a least square linear regression, the increase in record life expectancy averaging 2.4 years for 10 calendar years.

progress, and are not of foundational character. It is hence worthwhile to ask how taxpayers' money allocated for public science funding could be spent most efficiently.

In order to make a first inroad we investigate two large-scale endeavors of humanity. The first case study concerns the long-term impact of research and investments, in medicine and healthcare, on life expectancy over the last 170 years, asking the somewhat antipolar question: Why did the average life expectancy rise so slowly? The second example regards the advances in the reliability of short and medium term numerical weather forecasts since the 1950s. Weather dynamics has potentially chaotic regimes and the pace of progress in predictive meteorology may be limited by a complexity barrier resulting from systemic difficulties in predicting chaotic dynamical systems.

We find that measurable progress in S\&T is a highly sub-linear function of the invested resources, reflecting the law of diminishing returns well studied in economical contexts [2]. Some scientific insights can be achieved only through large collaborative projects, like the search for the Higgs Boson. Our results however show that small scientific endeavors do generically offer a higher potential for returns in terms of results per allocated funding.

Many natural systems investigated in the sciences are complex dynamical systems [3]. The brain in the neurosciences and the human genome in bioinformatics are examples from the realm of life. Short- and long term weather and climate evolution, many-body systems in condensed matter physics and elementary particle condensates are examples from the realm of physics. Complex systems are both difficult to understand and to investigate on a conceptual basis as well as to model and simulate numerically. These two difficulties impinge the pace of progress when investigating complex biological, or physical systems. We find the notion of a malleable complexity barrier to be a good visualization for the challenges confronting today's scientists. 


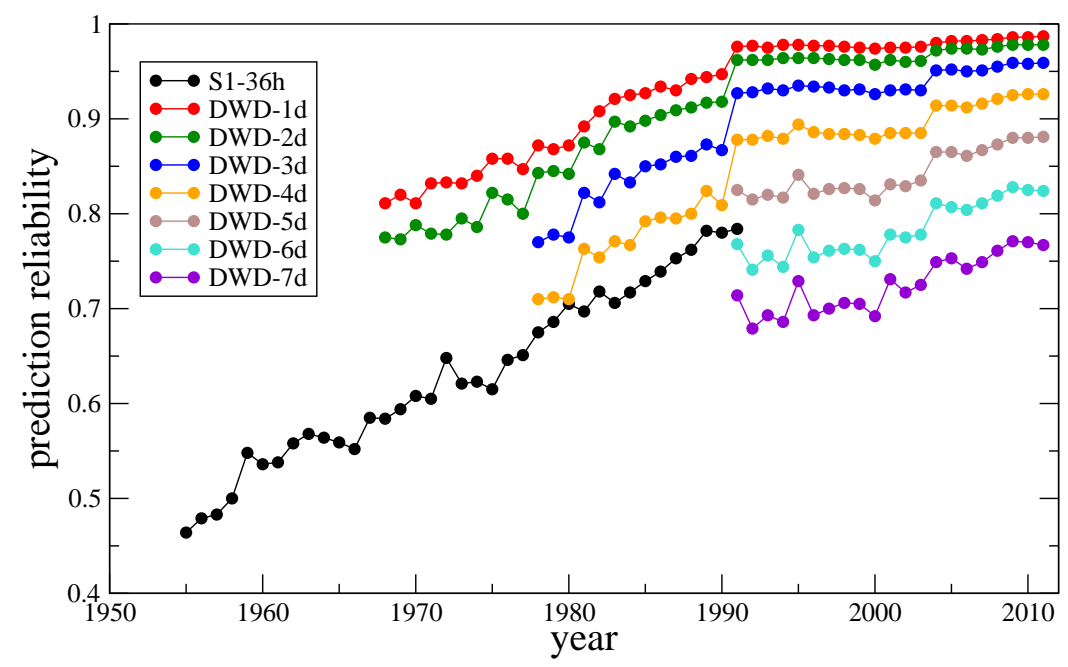

Figure 2: Shown are two distinct weather forecast reliability measures, the 500MB 36h (hour) S1 score of the NOAA [17] (black data), and the 1d-7d (days) $500 \mathrm{hPa}$ correlation coefficient of the DWD [15] (color data). Note that these two reliability scores are differently defined and cannot be compared directly on a quantitative basis.

\section{Record life expectancy}

Human life expectancy has seen a dramatic rise since the mid of the 18th century. On a global level one commonly considers the 'record life expectancy' as the life expectancy at birth of the country having the highest life expectancy worldwide. The record life expectancy has seen a strikingly linear growth for about 1.5 centuries, as shown in Fig. 1. It has been repeatedly predicted that this steady increase of human life expectancy would need to level off at a certain point, invoking biological limits. All these predicted limits have been broken hitherto without exception [4, 6]. This spectacular steady growth of the record life expectancy raises a series of interesting points.

Advances in healthcare and medicine will lead generically to raising levels of life expectancy. It remains however unclear which forces determine the magnitude of the observed rate, 2.4 years per every 10 calendar years, and how the observed growth rate depends on the overall amount of resources devoted [7]. Breakthroughs in research have been postulated on one hand to boost human lifespans rapidly [8, a putative natural limit, if existing [4, 9], should lead on the other side to a gradual levelling off. We have yet no definite answers to these foundational questions.

Longevity is a central issue in our culture and major efforts and resources are devoted by our societies towards increasing health levels and lifespan. Fig. 1 demonstrates that returns on investments have dramatically decreased during the last 1.5 centuries. The initial growth in life expectancy resulted from simple hygiene measures, followed by progress in immunology and antibiotics research. Lately, massive investments in pharmacology, technical medicine and bioinformatics have been necessary to keep up the steady linear advance in life expectancy. Relative progress has actually decreased in spite of these massive efforts, a linear increase relative to a base of 45 years is twice as large as a linear increase (with the same slope) relative to a base of 90 years.

Investment in medicine and healthcare have seen seen a roughly exponential increase since at 
least half a century [12, 13]. The driving forces behind these ever raising costs for healthcare and medicine are debated and could be rooted either in the desire to increase health and well-being quite generally or, more directly, in the quest to postpone death as far as possible. It has been argued, in this context, that the economic rational behind the ever raising levels of health spending lies in the fact that humans attribute an income elasticity well above unity to improvements of life expectancy, which seems to have psychologically a non-declining marginal utility [14. This argument indicates that the average life-expectancy is indeed a valid yardstick for measuring the overall advancement in health and medicine. The quantitative efficiency of S\&T research efforts in the healthcare sector is hence, as measured by the observed extension of human life expectancy, a highly sub-linear function of resources devoted. Returns on investment are seen to diminish rapidly in medicine and healthcare.

\section{Weather forecast reliability}

The dynamics of weather and climate for medium to long time scales is known to contain chaotic components 10. Indeed, one of the central models in the theory of chaotic and complex systems, the Lorenz model [1], is a hydrodynamic convection model. Medium to long term weather forecast is hence considered a challenge and massive investments in modeling, data acquisition and computational infrastructure have been made in order to achieve improved forecast performances.

A range of forecasting skill scores for numerical short to medium term weather prediction are evaluated continuously, in order to track the quality of daily weather predictions, by national and cross-national weather and climate agencies. In Fig. 2 the historical evolution of two distinct prediction reliability measures are shown, the first is the 1-7 day $500 \mathrm{hPa}$ forecast correlation coefficients of the DWD (Germany Weather Service) [15, 16. A value close to unity signals optimal forecasting, values below $60 \%$ corresponding to essentially useless predictions. The introduction of a new model in 1990 is reflected in the data. Also given in Fig. 2, for a longer-range perspective (1955-1991), is the S1 36 hour skill score of the NOAA (National Oceanic and Atmospheric Administration) [17, 18, which we normalized to the interval $[0,1]$. The $\mathrm{S} 1$ skill score is qualitatively different, containing gradients, from the $500 \mathrm{hPa}$ correlation coefficient [18, a direct comparison of the absolute values is not meaningful for these to weather forecasting reliability measures.

One of the key ingredients for numerical weather prediction, besides modeling and data acquisition [18, is computing power. Available computing power has seen an exponential growth over the last 60 years, Moore's law [19, 20], with a doubling period of about 1.5 years. The resulting advances in computational power has been about $10^{10}$ in 50 years. The computational facilities employed for numerical weather forecasting have seen corresponding increases [18, contributing to the observed improvements in weather forecasting skills.

In order to estimate the scaling between computational resources and forecasting skills quantitatively, we show in Fig. 3 the standardized forecasting error, (1 - reliability), corresponding to the remaining difference to optimal forecasting. The S1 skill score data is too distant from optimality, we hence focus on the DWD data for a systematic analysis. In Fig. 3 we have reploted the DWD data as $-\log (1-$ reliability $)$, using a trailing 3 -year average as a smoothing procedure. In order to compare the growth rates of prediction accuracy for different forecasting timescales we analyze the data presented in Fig. 3 using least square regressions. They fit the data reasonably well, indicating that the long-term growth of prediction accuracy is roughly exponential.

The time needed to double the relative accuracy, i.e. to half the the forecasting error $(1-$ reliability), grows systematically with increasing forecasting timespan (see the legend of Fig. 31). 


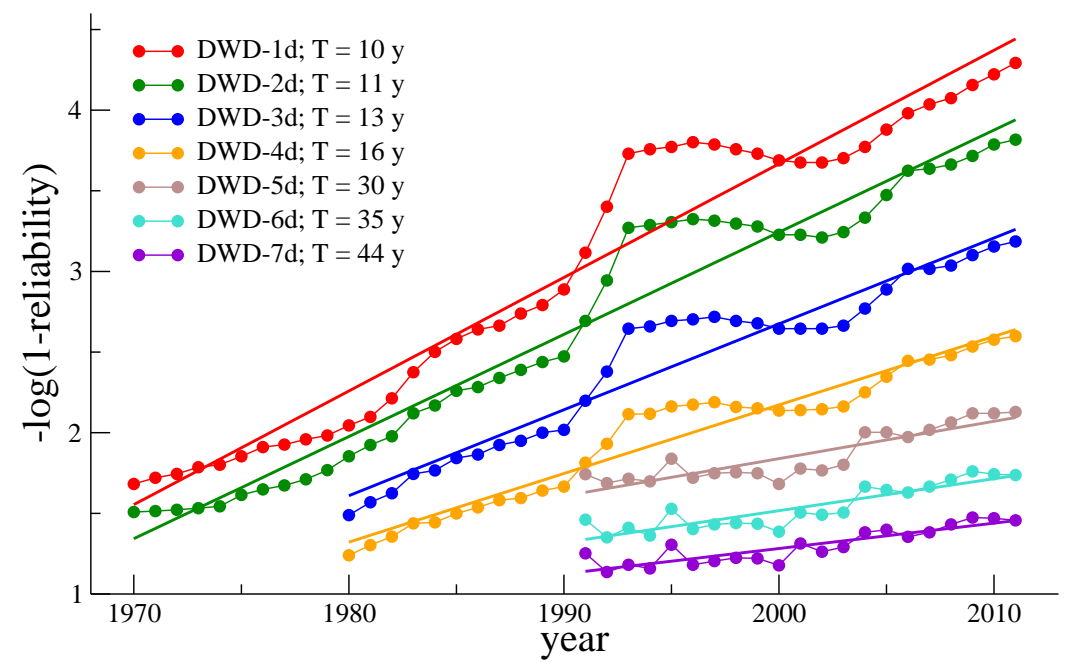

Figure 3: Linear-log plot of the DWD data (three years trailing average) of Fig. 2. The lines are least square regressions, the inverse slope in terms of the number $T$ of years needed to double the relative precision is given in the legend.

The accuracy of one-day weather predictions has doubled historically roughly every 10 years. Of the order of about 40 years seem to be necessary, on the other hand, for improving the reliability of seven day weather forecasting by a corresponding factor. Out of these results we conclude the following:

Firstly, the quantitative progress in weather prediction accuracy is a highly sub-linear function of committed computing resources. The accuracy scales roughly, with respect to the power $P_{c}$ of the computer facilities employed, as $\left(P_{c}^{1.5}\right)^{1 / 10}=P_{c}^{0.15}$ for one day forecasting and as $\left(P_{c}^{1.5}\right)^{1 / 40}=$ $P_{c}^{0.0375}$ for seven day predictions. The reliability of the skill scores is dependent additionally on advances in modeling and data acquisition, the respective functional relations of these dependencies is however beyond the scope of the present discussion.

Secondly, forecasting becomes rapidly more difficult with increasing prediction periods. Indeed it has been suggested that it may essentially be impossible to achieve useful forecasting reliabilities for 14-21 days in advance [21, at least with economically justifiable amounts of resources. This can be seen by plotting the weather forecasting scores as a function of forecasting period, as done in Fig. 4, where we have included also the T255L40 reliability score of the ECMWF (European Center for Medium-Range Weather Forecasts) from 2001 [21. Also shown in Fig. 4 are guides to the eye in the form of two-parameter least-square fits of the functional form

$$
1-\frac{t}{t+a \exp (-b t)}
$$

for $t=1,2, \ldots$ days, with adjustable parameters $a$ and $b$. This functional form captures the notion that it becomes progressively more difficult to achieve reliable forecasting with increasing prediction periods, the functional form of this increase in complexity has been assumed here to be exponential.

Generalizing these two observations we may relate the measured S\&T progress to the amount 


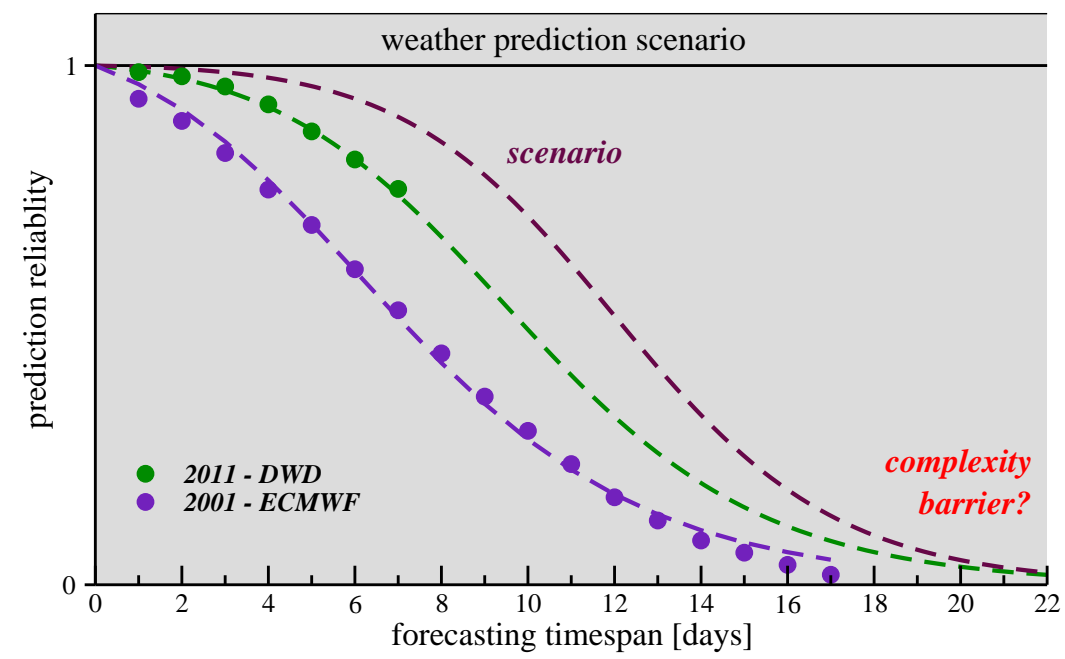

Figure 4: Forecasting reliability of the ECMWF (European Center for Medium-Range Weather Forecasts) T255L40 score from 2001 [21 (violet data), renormalized to [0,1], and of the 2011 DWD $500 \mathrm{hPa}$ score (see Fig. 2, green data). The dashed lines are two-parameter fits using Eq. (1), intended as guides to the eye. The brown dashed line represents a putative scenario for reliability scores achievable with further advancements.

of committed resources via the scaling relation

$$
\text { progress } \propto(\text { resources })^{\alpha},
$$

with a sub-linear scaling exponent $\alpha<1$. Our results, Figs. 3 and 4 indicates, that the scaling exponent $\alpha$ rapidly drops towards zero with increasing complexity of the task to be solved. We propose to use the term 'complexity barrier' for this phenomenon. For the case of the extension in human life expectancy, which is raising linearly, invested resources have increased roughly exponentially [12, 13, leading to a logarithmic relation, progress $\propto \log$ (resources). A log-relation corresponds to a vanishing scaling exponent, $\alpha \rightarrow 0$, indicating that increasing the average human life expectancy is a task of very high complexity.

\section{Entering the human factor}

The complexity barrier present in the context of short to medium term weather forecast is not hard. Progress is achievable when committing increasingly larger amounts of resources. The same holds for the complexity barrier present in the aging problem. Extending the average human life expectancy has been possible for the last 170 years by devoting strongly increasing amounts of resources to medicine and healthcare. The magnitude of the resources committed has been growing not only in absolute terms, but also as a fraction of national GNPs. The rational for the underlying collective decision of resource distribution is to be attributed to the human factor, progress in extending the human life span is highly valued.

We have discussed here only two examples, but we believe that progress in science, whenever it can be measured on a quantitative basis, is quite generically a strongly sublinear function $f(x)$ 
of the amount $x$ of resources devoted. Sublinear dependencies are concave and for any concave function $f(x)$ the total return $\sum_{i} f\left(x_{i}\right)$ is larger when splitting the total amount of resources $x$ into a series of subpackages of smaller sizes $x_{i}$,

$$
f(x)<\sum_{i} f\left(x_{i}\right), \quad \sum_{i} x_{i}=x
$$

It constitutes hence, for funding agencies, a substantially more efficient use of available resources to prioritize small to medium size projects, whenever feasible.

It is interesting to speculate, on a last note, whether the human factor influences the pace of progress in S\&T in an even more direct way. The human brain is well known to discount incoming information streams logarithmically, a relation known as the Weber-Fechner law [22, 23, 24]. This exponential data compression is necessary in order not to drown in the daily flood of sensory

impressions. It has been observed recently, that these neuropsychological constraints shape the statistics of global human data production in terms of publicly available data files in the Internet 25. The human factor is hence in evidence, at least in this particular aspect of human S\&T efforts, in the statistics of global public data generation. It is hence conceivable, as a matter of principle, that the pace of progress close to a complexity barrier is not only influenced by the overall amount of financial and physical resources committed, but also more directly by the neuropsychology of human thought processes.

I would like to thank Ulrich Achatz, George Craig, Ulrich Damrath, Peter Lynch, Detlev Majewski, Peter Nevir and Johannes Quaas for support collecting the meteorological skill scores, Roland Rau and James Vaupel for the life expectancy data.

\section{References}

[1] E. Pennisi ENCODE Project Writes Eulogy for Junk DNA. Science 337, 1159-1161 (2012).

[2] A. Marshall, Principles of economics. Cosimo Classics (2009).

[3] C. Gros, Complex and Adaptive Dynamical Systems, A Primer. Springer (2008); second edition 2010.

[4] J. Oeppen, J.W. Vaupel Broken Limits to Life Expectancy. Science 296, 1029-1031 (2002)

[5] Data since 2001 courtesy R. Rau and J. Vaupel. Source: Human Mortality Database (http://www.mortality.org).

[6] K.M. White, Longevity Advances in High-Income Countries, 1955-96. Population and Development Review, 28, 59-76 (2002).

[7] S. Tuljapurkar, N. Li, C. Boe, A universal pattern of mortality decline in the G7 countries. Nature 405, 789-792 (2000).

[8] A.D.N.J. De Grey, The foreseeability of real anti-aging medicine: focusing the debate. Experimental Gerontology 38, 927-934 (2003).

[9] J. Vijg, Y. Suh, Genetics of longevity and aging. Annual Review of Medicine 56, 193-212 (2005). 
[10] J. Shukla, Predictability in the midst of chaos: A scientific basis for climate forecasting. Science 282, 728-731 (1998).

[11] E.N. Lorenz, Deterministic nonperiodic flow. Journal of the Atmospheric Sciences, 20, 130-141 (1963).

[12] G.F. Anderson, J. Hurst, P.S. Hussey, M. Jee-Hughes, Health spending and outcomes: trends in OECD countries, 1960-1998. Health Affairs 19, 150-157 (2000).

[13] A. Sisko, C. Truffer, S. Smith, S. Keehan, J. Cylus, J.A. Poisal, M.K. Clemens, J. Lizonitz, Health spending projections through 2018: recession effects add uncertainty to the outlook. Health Affairs 28, w346-w357 (2009).

[14] R.E. Hall, C.I. Jones, The value of life and the rise in health spending. Quarterly Journal of Economics 122, 39-72 (2007).

[15] Data courtesy German Meteorological Service (DWD), Ulrich Damrath.

[16] A. Murphy, E. Epstein, Skill scores and correlation coefficients in model verification. Monthly Weather Review 117, 572-581 (1989).

[17] R.Y. Hiragano, The National Meteorological Center's Historical 36- (30-) Hour S1 Score Record. NOAA office note 389 (1992).

[18] P. Lynch, The origins of weather prediction and climate modeling. Journal of Computational Physics 277, 3431-3444 (2008).

[19] G.E. Moore Cramming more components onto integrated circuits. Proceedings of the IEEE 86 82-85 (1998).

[20] S.E. Thompson, S. Parthasarathy, Moore's law: the future of Si microelectronics. Materials Today 9, 20-25 (2006).

[21] A.J. Simmons, A. Hollingsworth, Some aspects of the improvement in skill of numerical weather prediction. Quarterly Journal of the Royal Meteorological Society, 128, 647-677 (2002).

[22] S. Hecht, The visual discrimination of intensity and the Weber-Fechner law. Journal of General Physiology 7 235-267 (1924).

[23] A. Nieder, E. K. Miller, Coding of Cognitive Magnitude: Compressed Scaling of Numerical Information in the Primate Prefrontal Cortex. Neuron 37 149-157 (2003).

[24] S. Dehaene, The neural basis of the Weber-Fechner law: a logarithmic mental number line. Trends in Cognitive Sciences 7 145-147 (2003).

[25] C. Gros, G. Kaczor, D. Markovic, Neuropsychological constraints to human data production on a global scale. European Physical Journal B 85, 28 (2012). 